\title{
CALIDAD DE VIDA EN MUJERES MENOPAUSICAS CON Y SIN TERAPIA DE REEMPLAZO HORMONAL
}

\author{
José Urdaneta M. MSc ${ }^{1}$, Maritza Cepeda de V. PhD ${ }^{2}$, Mery Guerra ${ }^{2}{ }^{2}$, Nasser \\ Baabel Z. PhD ${ }^{2}$, Alfi Contreras B. ${ }^{3}$
}

1 Universidad del Zulia, Posgrado de Obstetricia y Ginecología, Hospital Universitario de Maracaibo. ${ }^{2}$ Universidad del Zulia, Departamento de Ginecología y Obstetricia. ${ }^{3}$ Instituto Venezolano de los Seguros Sociales, Hospital Dr. Manuel Noriega Trigo.

\section{RESUMEN}

Antecedentes: El avance de la medicina ha incrementado la esperanza de vida al nacer y el crecimiento de la población mayor a 60 años, siendo cada vez mayor las mujeres en etapa de menopausia. Objetivo: Estudiar la calidad de vida (CV) en pacientes menopáusicas con y sin terapia de reemplazo hormonal. Método: Se estableció una investigación de tipo comparativa y aplicada, con diseño no experimental, contemporáneo transeccional y de campo, donde se incluyo una muestra de 200 pacientes con diagnóstico de menopausia. Se les aplicaron los cuestionario de CV SF-36 y el Menopause Rating Scale (MRS). Resultados: Hubo diferencias significativas $(p<0,05)$ en cuanto al dolor físico y altamente significativas $(p<0,01)$, respecto a los síntomas somáticos, psicológicos y urogenitales en las pacientes no tratadas vs las del grupo que recibió TRH. Conclusión: Las pacientes bajo TRH perciben una mayor CV específica respecto a las pacientes que no la reciben, presentando síntomas somáticos o psicológicos menos severos.

\section{PALABRAS CLAVE: Calidad de vida, menopausia, terapia de reemplazo hormonal}

\section{SUMMARY}

Background: The medicine's advance has increased the life expectancy when being born and the growth of the greater population of 60 years, being increasing the women in menopause stage. Objective: To study the quality of life (QL) in menopause's women with and without hormonal therapy. Method: An investigation of comparative and applied type was settle down, with nonexperimental, contemporary transeccional and field design, where was included a sample of 200 patients with diagnosis of menopause. To these patients was be applied a questionnaire of QL, SF-36 and the Menopause Rating Scale (MRS). Results: It was a significant differences $(p<0.05)$ in the presence of physical pain and highly significant differences $(p<0.01)$ respect to the somatic, psychological and urogenitals symptoms in the nontreated patients when comparing them with those who received TRH. Conclusion: Patient under TRH perceives a greater specific QL with respect to the patients who do not receive it, presenting less severe somatic or psychological symptoms.

KEY WORDS: Life's quality, menopause, hormonal replacement therapy 


\section{INTRODUCCIÓN}

El siglo XX ha sido decisivo para el desarrollo de los diferentes ámbitos de la medicina, los grandes adelantos alcanzados han permitido incrementar la esperanza de vida al nacer y modificar las pirámides poblacionales de una gran cantidad de países, siendo mayor la cantidad de personas mayores de 60 años, sobretodo mujeres (1); Por consecuencia, cada día serán más mujeres que estarán viviendo y en muchos casos padeciendo la menopausia.

Asimismo, se ha producido un cambio de paradigma en la concepción del estado de salud, incorporando una visión holística e integral del ser humano; surgiendo así el concepto de la calidad de vida relacionada a la salud (CVRS), el cual viene a constituir un nuevo indicador para la valoración de las consecuencias de las enfermedades sobre la percepción de salud de cada individuo, así como de los resultados de las diferentes intervenciones médicas en el manejo del proceso salud-enfermedad. Según el criterio de la Organización Mundial de la Salud la CVRS se refiere a la "percepción del individuo de su posición en la vida, en el contexto de la cultura y sistema de valores en los que vive y en relación con sus objetivos, expectativas, estándares y preocupaciones" (2). Este constructo pretende valorar aspectos de la enfermedad relacionados con la vida diaria del paciente y de que modo se ve afectada por la patología o por la medicación concomitante, abarcando por lo menos cuatro dimensiones a saber: física, funcional, psicológica y social. De modo que, cada una de estas dimensiones puede ser afectada a mediano y largo plazo por la enfermedad, y a corto plazo por la medicación administrada (3).

La CVRS constituye un indicador caracterizado por tomar en cuenta la perspectiva del individuo, dando cuenta de una subjetividad no evaluada por la mayoría del resto de los indicadores utilizados en salud, siendo esta única cualidad la que le asigna un lugar que junto con la información clínica puede ser muy útil en la toma de decisiones médicas (4). De esta manera, las tradicionales medidas de mortalidad y morbilidad están dando paso a una nueva forma de valorar los resultados de las enfermedades e intervenciones médicas, comparando unas con otras; y en esta línea, la meta de la atención en salud se está orientando no sólo a la eliminación de la patología, sino fundamentalmente a la mejora de la CV del paciente (5).

En la región de las Américas, así como en el resto del mundo, el desarrollo ha originado un aumento en la esperanza de vida al nacer, descenso de las tasas de mortalidad, incremento en el creci- miento de la población mayor de 60 años y de la incidencia de enfermedades crónicas; sobretodo en el sexo femenino, quienes han demostrado ser más longevas que los hombres (1). El envejecimiento constituye un proceso natural e inevitable que en la mujer se acompaña de un estado fisiológico particular: la menopausia. En este periodo ocurre el cese de la función hormonal ovárica, marcando el final de la vida reproductiva de la mujer. Alrededor de la menopausia, cuando comienza a disminuir la producción hormonal ovárica, posterior al cese de las menstruaciones, la mujer atraviesa un periodo denominado climaterio, donde empieza a presentar signos y síntomas relacionados fundamentalmente con la carencia hormonal.

La menopausia se entiende como el término médico que designa la fecha de la última menstruación, etimológicamente proviene del griego meno (mes) y pausia que significa cese, siendo los 50 años la edad promedio para que ocurra, con límites que oscilan entre los 42 y 56 años (6). En Venezuela el promedio es de 48,7 años para la menopausia natural y de 43,3 años para la menopausia artificial (7).

La menopausia representa una etapa de adaptación y transición a una nueva situación biológica que conlleva la pérdida de la capacidad reproductiva, su instauración tiene un carácter progresivo, afectando a todas las mujeres sanas, no asociándose con la génesis directa de ninguna noxa, no existiendo ninguna base científica que permita considerarla como una entidad patológica (8). Tanto el incremento de la población en la tercera edad como el aumento de la esperanza de vida, han influido en un mejor entendimiento de la menopausia por parte de los profesionales sanitarios produciendo una mejora en el enfoque de los tratamientos y en la prevención de sus consecuencias (6).

La menopausia, por su parte, no debe ser sinónimo de deterioro, por el contrario debe ser vista como una pausa para reflexionar sobre el quehacer como mujeres, profesionales, o madres, constituye una transición social, a veces difícil u dolorosa, pero también de apertura y oportunidades en distintos ámbitos (9). Sin embargo, esta trae consigo una sintomatología específica que interfiere en la CV de la mujer, este concepto durante esta etapa de la vida abarca numerosas facetas de la vida que incluyen además de la salud, una perspectiva psíquica y sociológica, con un alto componente subjetivo (10).

En el climaterio y menopausia se producen una gran variedad de manifestaciones clínicas que incluyen síntomas vasomotores, psíquicos, atrofia urogenital, enfermedades cardiovasculares, osteoporosis 
y mayor riesgo de enfermedad de Alzheimer, manifestaciones que tendrían su efecto en la CV de la mujer (11). Por tanto, no es suficiente con que el médico identifique la presencia de bochornos u otro síntoma, sino que interesa la apreciación subjetiva de la mujer quien dirá si ve afectado o no su bienestar por esa molestia, si estos cambios afectan su capacidad laboral, su capacidad de relacionarse con diferentes personas, entre otras (12).

Álvarez y cols (13), establecen que el climaterio y la menopausia pueden alterar la calidad de vida de la mujer, produciendo secuelas por el incremento en el riesgo de sufrir enfermedad isquémica cardiovascular y osteoporosis. En este periodo de la existencia se puede alterar la calidad de vida de la mujer debido a que las manifestaciones vasomotoras que lo acompañan, caracterizadas por bochornos, rubor y sudoración a predominio nocturno, pueden llegar a ser muy molestas. Asimismo se acentúa el insomnio que acompaña a las alteraciones neuro-psíquicas, produciendo irritabilidad, cambios de carácter, pérdida de la concentración o memoria, y disminución o ausencia de la libido $(13,14,15)$.

La gama de manifestaciones en la esfera psicológica comprenden síntomas como humor depresivo irritabilidad, labilidad afectiva, falta de concentración; así como pérdida de la libido y otros problemas sexuales que también pueden conducir a la depresión (14). También, se ha observado que entre $33-51 \%$ de las mujeres de edad mediana presentan trastornos del sueño al tiempo que transcurren por la menopausia; estos pueden resultar de cambios hormonales, síntomas vasomotores o factores psicológicos (15). Los cambios físicos que acompañan al climaterio deben tener escasos efectos sobre el funcionamiento sexual, sin embargo aspectos psicológicos propios de este momento vital, la ausencia o mala interacción con la pareja y las estereotipias culturales pueden llegar a determinar la presencia de serias disfunciones sexuales (6).

Es así como al encontrarse en el período climatérico, la mujer y el médico tratante se enfrenta con varias decisiones importantes, entre las cuales, una de las más significativas, es si se debe comenzar o no el tratamiento hormonal. Al respecto existen muchas interrogantes que pueden surgir: ¿cuánto tiempo tomarlo?, ¿qué efectos adversos pudiese acarrear su utilización?, ¿qué tipo de terapia de reemplazo utilizar?, ¿superarán los riesgos los beneficios de la terapia?, entre muchas más dudas e inquietudes que pudiesen surgir.

El tratamiento con estrógenos ha estado disponible para la mujer posmenopáusica por más de 60 años, proveyendo alivio de la sintomatología vasomotora, de la atrofia vaginal y previniendo la osteoporosis. Sin embargo, a pesar de décadas de uso y de infinidad de investigaciones, el rol de los estrógenos como terapia única en la prevención de enfermedades crónicas en estas mujeres permanece incierto (16).

En este sentido, a comienzos de este nuevo siglo se hizo público uno de los estudios que mayor interés ha despertado en la prensa, tanto científica como profana: The Women's Health Initiative (WHI), donde se concluía contundentemente que los riesgos superaban a los beneficios del tratamiento hormonal; causando su publicación un profundo impacto en la actitud de pacientes y médicos, especialmente sobre el uso o no de terapia hormonal (13).

Este y otros ensayos clínicos han asumido que los riesgos para la salud están asociados con el uso combinado de hormonas, sin embargo, poco se conoce acerca del efecto de la terapia hormonal sobre la calidad de de vida relacionada con la salud. Al respecto, Hays y cols (17), exponen que el uso de combinaciones de estrógenos más progestágenos no tenía un efecto deletéreo clínicamente significativo sobre cualquiera de los aspectos de la salud evaluados en el estudio WHI; asimismo encontró que los beneficios sobre la calidad de vida eran mayores cuando se usaban por largo plazo debido a que durante el primer año de su utilización pudiesen pasar desapercibidos.

Este estudio ha originado grandes controversias, sin haberse podido establecer si los beneficios encontrados en los ensayos contrarios al WHI compensan o no los riesgos de ataque cardíaco, accidente cerebrovascular, coágulos sanguíneos y cáncer de mama asociados con la terapia hormonal combinada. En virtud de esta panorámica, siendo finalmente cada mujer quien obtiene los beneficios y sufre las consecuencias de cualquier tratamiento, su decisión de recibir terapia de reemplazo hormonal $u$ otros tratamientos alternativos en la menopausia natural debe ser respetada (9).

Desterrar cualquier connotación de enfermedad o de patología ligada al acontecimiento fisiológico de la menopausia, es probablemente uno de los aspectos más importantes a desarrollar desde cualquier ámbito de la salud y que más puede contribuir a mejorar la CV durante esta etapa vital. En este sentido, es oportuno señalar que la expectativa de vida actual de la mujer venezolana es de 73,1 años, por lo que aproximadamente un tercio de su existencia transcurrirá en la menopausia, de allí la importancia de propiciar una vida satisfactoria para la mujer (13).

Existen múltiples instrumentos para evaluar la 
CVRS, los cuales se emplean con el propósito de discriminación entre sujetos afectados, predecir el pronóstico o resultado de una intervención y evaluar los posibles cambios en el tiempo, que permitiría valorar la supervivencia frente a tratamientos o intervenciones (18). Los cuestionarios de CV no sustituyen a las evaluaciones sintomáticas, analíticas o morfológicas, sino que las complementan, introduciendo algo tan trascendente como la visión del propio paciente sobre su percepción de salud (19).

Estos instrumentos han sido clasificados en genéricos y específicos, los primeros se emplean para comparar las diferentes poblaciones y padecimientos, mientras que los específicos se basan en las características específicas de una determinada patología (5). Dentro de los cuestionarios genéricos, uno de los más utilizados es el cuestionario SF-36, desarrollado en los Estados Unidos, el cual proporciona un perfil del estado de salud y es aplicable tanto a los pacientes como a la población general. Su contenido, que incluye tanto salud física como mental consta de 36 ítems agrupados en 8 dimensiones: (a) función física, (b) rol físico, (c) dolor corporal, (d) salud general, (e) vitalidad, (f) función social, (h) rol emocional y (i) salud mental; estos ítems detectan tanto estados positivos como negativos de la salud y permiten generar un índice global que tiene un recorrido desde 0 (el peor estado de salud para esa dimensión) hasta 100 (el mejor estado de salud) $(18,20)$. Algunas versiones, han adicionado un ítem de transición que pregunta sobre el cambio en el estado de salud general respecto al año anterior; asimismo existe una versión en español que muestra coincidencia completa con la versión original, alta equivalencia con los valores originales y reproducibilidad aceptable, aunque menor que la original (20).

Para valorar la CV en el climaterio y menopausia se deben considerar los fenómenos que ocurren tanto en el área física como en las áreas psicológica, sexual, social y familiar de la mujer. Tradicionalmente, la evaluación de las consecuencias que la menopausia tiene sobre la mujer se ha hecho a partir de escalas clínicas; las cuales han pretendido proporcionar una cuantificación numérica que pueda ser usada para la evaluación de la eficacia de las intervenciones médicas (21). Existen en la literatura diferentes propuestas como: el Women's Health Questionnare (WHQ), la Escala de Greene, la Menopausal Synthoms List, Menopause Rating Scale (MRS), la Utian Menopause Quality of Life Scale (UMQLS), la Menopausic Specific Quality of Life (MEQOL); una versión en lengua castellana del MEQOL, el Cuestionario MENCAV, el cuestionario de calidad de vida incluido en el PAIMC y recientemente la Escala Cervantes, entre otros (5,18-23).

En la presente investigación se utilizó la escala "Menopause Rating Scale" (MRS), que consiste en un cuestionario estandarizado de autoevaluación integrado por 11 ítems que miden síntomas somáticos, psicológicos y urogenitales (22). Este es un instrumento bien definido de autoevaluación de los síntomas menopáusicos que permite de una manera práctica y relativamente rápida evaluar el impacto de cualquier intervención médica con relación a diferentes aspectos de la CVRS, asimismo, puede medir la mejoría en los pacientes que comienzan con la terapéutica. Constituye un excelente instrumento de medición de CV específicamente desarrollado para ser aplicado en el climaterio, y que a diferencia de otras escalas consta de un cuestionario breve que debe ser contestado directamente por la encuestada, sin la intervención del sesgo de encuestadores (23).

Esta escala contiene cinco opciones de respuesta que representan cinco grados de severidad: asintomático (0 puntos), leve (1 punto), moderado (2 puntos), severo (3 puntos) e intenso (4 puntos); el puntaje total de MRS oscila entre 0 (asintomática) y 44 (grado máximo de disturbio por síntomas menopáusicos) (24). Heinemann y cols (25), expresan que diversos estudios efectuados en diferentes países han permitido validar esta escala y estandarizar la intensidad de la sintomatología en asintomática o escasa (0-4 puntos), leve (5-8 puntos), moderada (9-15 puntos), y severa (más de 16 puntos). También se han establecidos diferentes puntos de cortes dentro de esta misma escala para los diferentes dominios: (a) 1 punto o menos, 2 a 3 puntos, 4 a 6 puntos y más de 7 puntos para el dominio psicológico; (b) 2 puntos o menos, 3 a 4 puntos, 5 a 8 puntos y más de 9 puntos para el dominio somático; y (c) o punto, 1 punto, 2 a 3 puntos y 4 puntos o más para los síntomas urogenitales.

En la actualidad esta escala se encuentra disponible en 25 idiomas y ha sido validada continuamente en diferentes países, lo cual ha permitido establecer valores estándares para diferentes poblaciones (24-26). Así pues, para Latinoamérica los promedios establecidos son: (a) 10,4 $\pm 8,8 \mathrm{DE}$ para el puntaje total, (b) 4,1 $\pm 3,6 \mathrm{DE}$ para el dominio somático, (c) 4,9 \pm 4,5 DE para la esfera psicológica, y (d) 1,4 $\pm 2,2$ DE para los síntomas urogenitales (26). Cabe destacar, que en Venezuela, para la fecha de esta investigación, los instrumentos de evaluación funcional y de calidad de vida aún no han sido diseñados, motivo por el cual se han adoptado los instrumentos de otros países.

El objetivo de esta investigación es comparar la 
CV de las pacientes menopáusicas atendidas en la Maternidad "Dr. Armando Castillo Plaza", sede de la División de Ginecología y Obstetricia del Hospital Universitario de Maracaibo, principal centro de salud del occidente venezolano, de manera que permitiese conocer la percepción de estas pacientes sobre la menopausia y su relación con la toma o no de la terapia de reemplazo hormonal (TRH). Para ello se analizó tanto la CV genérica como específica de las mujeres menopáusicas que reciben o no la terapia hormonal.

\section{MATERIAL Y MÉTODOS}

De acuerdo al problema planteado, referido por la CV en mujeres menopáusicas con o sin $\mathrm{TRH}, \mathrm{y}$ en función de los objetivos propuestos se puede afirmar que esta investigación fue básicamente de tipo comparativa y aplicada; con un diseño no experimental, de campo, contemporáneo transeccional y univariable.

En la presente investigación las unidades de análisis objeto de observación o estudio estaban representadas por la totalidad de pacientes menopáusicas atendidas en la consulta de Ginecología de la Maternidad "Dr. Armando Castillo Plaza". Importa destacar, que la población de estudio en el presente caso, estuvo representada por 400 pacientes con diagnóstico de menopausia, las cuales tuvieron su primera consulta en esta institución durante el año 2007, de acuerdo con datos obtenidos del departamento de historia y registros médicos. Para determinar el tamaño de la muestra de la población que se estudió, con características finitas, con un nivel de confianza del $95 \%$, a dos sigmas y con un error de $5 \%$, se aplicó la fórmula para poblaciones finitas, resultando el tamaño global en 200 menopáusicas, numero de pacientes que se distribuyeron en dos grupos de 100 sujetos cada uno dependiendo del uso o no de la TRH.

La selección de las unidades de la muestra se realizó de manera intencionada, tomándose a las pacientes con diagnóstico de menopausia y separándolas en estos dos grupos, dependiendo de si cumplían con los siguientes criterios: (a) ausencia de menstruaciones por más de un año, (b) edad mayor de 45 años y menor de 60 años, (c) deseo voluntario de participar en la investigación, (d) no presentar contraindicaciones absolutas para la TRH y (e) no ser analfabeta o presentar discapacidad visual.

Dada la naturaleza del estudio y en función de los objetivos propuestos, se tomó como técnica para la recolección de los datos, la observación indirecta; procediéndose a aplicarla mediante la ejecución de dos cuestionarios auto-administrados para la valoración de la CV, uno genérico y otro específico para la población en etapa de menopausia. El primer instrumento aplicado fue el Cuestionario de Salud SF-36, en su versión española 1.4; mientras que el instrumento específico seleccionado fue la versión en español de la escala MRS (Anexo 1). Asimismo, se utilizó la ficha de trabajo o cuadro de observación como instrumento complementario con el fin de recopilar información sobre algunas otras variables intervinientes, como lo son: (a) edad, (b) edad de la menopausia, (c) utilización de la TRH, (d) tipo de TRH empleada, (e) años de consumo de la $\mathrm{TRH}, \mathrm{y}$ (f) antecedentes personales.

Para la ejecución de esta investigación, se procedió a seleccionar en la consulta ginecológica a las pacientes menopáusicas de acuerdo con los criterios previamente establecidos, dividiéndolas en dos grupos pareados de acuerdo con el uso o no de la TRH. A cada paciente, se le entregaron los dos instrumentos seleccionados, previa explicación de los mismos, y se registraron los siguientes datos: edad cronológica, edad de la menopausia (definida para este estudio como un año de amenorrea y/o $\mathrm{FSH}>40 \mathrm{mUI} / \mathrm{mL}$ ), la utilización de $\mathrm{TRH}$, el tipo de terapéutica y el tiempo de uso, así como antecedentes personales de histerectomía, obesidad, hipertensión arterial, diabetes mellitus, incontinencia urinaria, prolapso genital, osteopenia u osteoporosis.

Los datos que se generaron de la aplicación de estos instrumentos fueron organizados y registrados en una base de datos. Posteriormente, mediante el Paquete Estadístico para Ciencias Sociales (SPSS), versión 11.0.1, se realizaron el cálculo de los índices provenientes de las escalas correspondientes; la sintomatología climatérica en ambos grupos se expreso mediante medidas de tendencia central: medias y desviación estándar (DE) y para realizar la comparación de los resultados obtenidos entre los dos grupos de pacientes evaluadas, se utilizó la prueba $U$ de Mann-Whitney para grupos independientes según distribución de edades y variables estudiadas.

\section{RESULTADOS}

El análisis descriptivo de algunas de las principales características presentes en las pacientes menopáusicas de ambos grupos se presenta en la Tabla I. La edad cronológica de las pacientes del grupo sin tratamiento evidenció un promedio de 55 años (rango: 50 y 59 años), y en el grupo que recibía tratamiento la edad promedio fue de 54 años (rango: 51 y 58 años), resaltándose la homogeneidad en las 
Tabla I

CARACTERIZACIÓN DE LA MUESTRA

\begin{tabular}{lcccc}
\hline $\begin{array}{l}\text { Análisis/ } \\
\text { Alternativas }\end{array}$ & $\begin{array}{c}\text { Pacientes no } \\
\text { tratadas } \\
\mathrm{X} \pm \mathrm{DE}\end{array}$ & $\begin{array}{c}\text { Pacientes } \\
\text { tratadas } \\
\mathrm{X} \pm \mathrm{DE}\end{array}$ & $\begin{array}{c}\text { Distribución con } \\
\text { tratamiento } \\
(\%)\end{array}$ & $\begin{array}{c}\text { Distribución sin } \\
\text { tratamiento } \\
(\%)\end{array}$ \\
\hline Edad & $55,00 \pm 4,51$ & $54,52 \pm 3,87$ & -- & -- \\
Edad de menopausia & $47,76 \pm 5,78$ & $48,32 \pm 2,82$ & -- & -- \\
Estrógenos equinos & -- & -- & 16,0 & -- \\
17 B Estradiol & -- & -- & 8,0 & -- \\
Estrógenos progestágenos & -- & -- & 8,0 & -- \\
Estrógenos transdérmicos & -- & -- & 12,0 & -- \\
Tibolona & -- & -- & 32,0 & -- \\
Estrógenos parenterales & -- & -- & 8,0 & -- \\
Fito-estrógenos & -- & -- & 12,0 & -- \\
Tiempo & -- & -- & -- \\
Histerectomía & -- & -- & 48,0 & 20,0 \\
Obesidad & -- & -- & 8,0 & 40,0 \\
HTA & -- & -- & 28,0 & 48,0 \\
Diabetes melitus & -- & -- & 8,0 & 4,0 \\
Dislipidemia & -- & & 12,0 & 28,0 \\
\hline
\end{tabular}

edades entre ambos grupos. La edad de aparición de la menopausia en el grupo sin tratamiento fue a los 48 años igual que en el grupo tratado.

Asimismo, el tratamiento prescrito más frecuentemente fue la tibolona en el $32 \%$ de la población estudiada, seguido por el uso de estrógenos equinos en el $16 \%$, estrógenos transdermicos y fitoestrogenos en el $12 \%$ cada uno respectivamente. Las terapéuticas menos empleadas el 17-beta estradiol, las combinaciones de estrógenos más progestágenos y los estrógenos parenterales. El uso de estos tratamientos en las pacientes fue en promedio de 3 años considerando un rango entre 2 y 4 años. Se evaluaron los antecedentes personales de las pacientes en ambos grupos evidenciándose que en el grupo de las pacientes tratadas el $48 \%$ tenían histerectomía, el $28 \%$ eran hipertensas, el $12 \%$ tenían antecedentes de dislipidemia y el $8 \%$ eran obesas o diabéticas (Tabla I).

El análisis de los indicadores evaluados en uno de los instrumentos seleccionados, el SF-36 evidenció medias y su respectiva DE por ítems, tanto en el grupo de pacientes menopáusicas con terapia hormonal como al grupo de pacientes sin tratamiento. Los resultados obtenidos del análisis la salud física en su indicador función física, en las pacientes del estudio evidenció que la alternativa 3 (No, no me limita nada) predominó en el grupo de pacientes tratadas con TRH y el grupo no tratadas la alternativa 2 ( $\mathrm{Si}$, me limita un poco), el rol físico se evaluó en ambos grupos donde la alternativa predominante fue la 2 (No), lo que permite afirmar que no hubo limitación alguna de actividad física en las pacientes. El dolor corporal si mostró diferencias entre grupos, en la muestra sin tratamiento las alternativas predominantes fueron 2 (Un poco) y 3 (Regular), y en el grupo tratado fueron la alternativas 1 (Nada) y 2 (Un poco), lo que indica que las que no reciben tratamiento presentan dolor corporal en alguna parte del cuerpo y este ocasiona interferencias para la realización de trabajos. También se evaluó en ambos grupos la salud general observándose que las alternativas de respuesta estuvieron dispersas entre ítems, en el grupo no tratado, donde las pacientes afirmaron que no se ponen enfermas más fácil que otras personas, no saben si están sanas como cualquiera, consideran que su salud no empeorara y que su salud es excelente; mientras que el grupo que recibe tratamiento respondió que no se ponen enfermas más fácil que otras personas, están sanas como cualquiera, consideran que su salud no empeorara y que su salud es excelente (Tablas II y III).

La salud mental también fue evaluada a través de sus indicadores y se evidencio que el rol emocional en las pacientes tratadas y no tratadas, mostró una tendencia mayor a la alternativa 2 (No), afirmando que los problemas emocionales no le afectan en su día a día, la actividad social de las pacientes tratadas tampoco se ve afectada, con una 
Tabla II

ANÁLISIS DESCRIPTIVO DE LA DIMENSIÓN CALIDAD DE VIDA GENÉRICA: SUB-DIMENSIÓN COMPONENTE FÍSICO EN PACIENTES TRATADAS

\begin{tabular}{|c|c|c|c|c|c|c|c|c|}
\hline \multirow[t]{2}{*}{ Escala de salud } & \multirow{2}{*}{$\begin{array}{c}\text { Contenido requerido } \\
\text { del ítems }\end{array}$} & \multirow[t]{2}{*}{$X \pm D E$} & \multicolumn{6}{|c|}{ Distribución porcentual del ítems } \\
\hline & & & 1 & 2 & 3 & 4 & 5 & 6 \\
\hline \multirow{10}{*}{ Función física } & Actividad intensa & $2,49 \pm 0,68$ & 11 & 29 & 60 & -- & -- & -- \\
\hline & Actividad moderada & $2,73 \pm 0,44$ & -- & 27 & 73 & -- & -- & -- \\
\hline & $\begin{array}{l}\text { Levantar o llevar } \\
\text { bolsas de compra }\end{array}$ & $2,72 \pm 0,45$ & -- & 28 & 72 & -- & -- & -- \\
\hline & $\begin{array}{l}\text { Subir varios pisos } \\
\text { de escaleras }\end{array}$ & $2,59 \pm 0,66$ & 10 & 21 & 69 & -- & -- & -- \\
\hline & $\begin{array}{l}\text { Subir un solo piso } \\
\text { por escaleras }\end{array}$ & $2,80 \pm 0,40$ & -- & 20 & 80 & -- & -- & -- \\
\hline & $\begin{array}{l}\text { Se limita para } \\
\text { arrodillarse }\end{array}$ & $2,57 \pm 0,60$ & 6 & 31 & 63 & -- & -- & -- \\
\hline & $\begin{array}{l}\text { Se limita caminar más } \\
\text { de un kilómetro }\end{array}$ & $2,64 \pm 0,54$ & 3 & 30 & 67 & -- & -- & -- \\
\hline & $\begin{array}{l}\text { Se limita caminar } \\
\text { varias manzanas }\end{array}$ & $2,77 \pm 0,48$ & 9 & 17 & 80 & -- & -- & -- \\
\hline & $\begin{array}{l}\text { Se limita caminar unos } \\
100 \text { metros }\end{array}$ & $2,96 \pm 0,19$ & -- & 4 & 96 & -- & -- & -- \\
\hline & $\begin{array}{l}\text { Se limita para bañarse } \\
\text { o vestirse }\end{array}$ & $2,92 \pm 0,27$ & -- & 8 & 92 & -- & -- & -- \\
\hline \multirow{5}{*}{ Rol físico } & $\begin{array}{l}\text { Menor tiempo } \\
\text { dedicado al trabajo }\end{array}$ & $1,86 \pm 0,34$ & 14 & 86 & -- & -- & -- & -- \\
\hline & $\begin{array}{l}\text { Hacer menos } \\
\text { actividades que las }\end{array}$ & $1,82 \pm 0,38$ & 18 & 82 & -- & -- & -- & -- \\
\hline & deseadas & & & & & & & \\
\hline & $\begin{array}{l}\text { Limitación en el tipo } \\
\text { de trabajo o actividad }\end{array}$ & $1,89 \pm 0,31$ & 11 & 89 & -- & -- & -- & -- \\
\hline & $\begin{array}{l}\text { Dificultades para hacer } \\
\text { el trabajo }\end{array}$ & $1,79 \pm 0,40$ & 21 & 79 & -- & -- & -- & -- \\
\hline
\end{tabular}

$\begin{array}{cccccc}1,76 \pm 1,10 & 56 & 27 & 6 & 7 & 4 \\ 1,45 \pm 0,86 & 76 & 7 & 13 & 4 & --\end{array}$

Dolor en alguna parte

\section{del cuerpo las últimas 4 semanas Interferencia del dolor para la realización de trabajos \\ Dolor corporal}




\section{Tabla III}

ANÁLISIS DESCRIPTIVO DE LA DIMENSIÓN CALIDAD DE VIDA GENÉRICA: SUB-DIMENSIÓN COMPONENTE FÍSICO EN PACIENTES NO TRATADAS

\begin{tabular}{|c|c|c|c|c|c|c|c|c|}
\hline \multirow{2}{*}{$\begin{array}{l}\text { Escala } \\
\text { de salud }\end{array}$} & \multirow{2}{*}{$\begin{array}{c}\text { Contenido requerido } \\
\text { del ítems }\end{array}$} & \multirow[t]{2}{*}{$\mathrm{X} \pm \mathrm{DE}$} & \multicolumn{6}{|c|}{ Distribución porcentual del ítems } \\
\hline & & & 1 & 2 & 3 & 4 & 5 & 6 \\
\hline \multirow{10}{*}{ Función física } & Actividad intensa & $2,05 \pm 0,74$ & 25 & 45 & 30 & -- & -- & -- \\
\hline & Actividad moderada & $2,31 \pm 0,69$ & 13 & 43 & 44 & -- & -- & -- \\
\hline & $\begin{array}{l}\text { Levantar o llevar } \\
\text { bolsas de compra }\end{array}$ & $2,36 \pm 0,65$ & 10 & 44 & 46 & -- & -- & -- \\
\hline & $\begin{array}{l}\text { Subir varios pisos } \\
\text { de escaleras }\end{array}$ & $2,36 \pm 0,70$ & 13 & 38 & 49 & -- & -- & -- \\
\hline & $\begin{array}{l}\text { Subir un solo piso } \\
\text { por escaleras }\end{array}$ & $2,50 \pm 0,73$ & 14 & 22 & 64 & -- & -- & -- \\
\hline & $\begin{array}{l}\text { Se limita para } \\
\text { arrodillarse }\end{array}$ & $2,30 \pm 0,75$ & 18 & 34 & 48 & -- & -- & -- \\
\hline & $\begin{array}{l}\text { Se limita caminar } \\
\text { más de un kilómetro }\end{array}$ & $2,37 \pm 0,67$ & 11 & 41 & 48 & -- & -- & -- \\
\hline & $\begin{array}{l}\text { Se limita caminar } \\
\text { varias manzanas }\end{array}$ & $2,42 \pm 0,75$ & 16 & 26 & 58 & -- & -- & -- \\
\hline & $\begin{array}{l}\text { Se limita caminar } \\
\text { unos } 100 \text { metros }\end{array}$ & $2,57 \pm 0,68$ & 11 & 21 & 68 & -- & -- & -- \\
\hline & $\begin{array}{l}\text { Se limita para } \\
\text { bañarse o vestirse }\end{array}$ & $2,78 \pm 0,48$ & 3 & 16 & 81 & -- & -- & -- \\
\hline \multirow{4}{*}{ Rol físico } & $\begin{array}{l}\text { Menor tiempo } \\
\text { dedicado al trabajo }\end{array}$ & $1,55 \pm 0,50$ & 45 & 55 & -- & -- & -- & -- \\
\hline & $\begin{array}{l}\text { Hacer menos } \\
\text { actividades que } \\
\text { las deseadas }\end{array}$ & $1,55 \pm 0,50$ & 45 & 55 & -- & -- & -- & -- \\
\hline & $\begin{array}{l}\text { Limitación en el tipo } \\
\text { de trabajo o actividad }\end{array}$ & $1,47 \pm 0,50$ & 53 & 47 & -- & -- & -- & -- \\
\hline & $\begin{array}{l}\text { Dificultades para } \\
\text { hacer el trabajo }\end{array}$ & $1,50 \pm 0,50$ & 50 & 50 & -- & -- & -- & -- \\
\hline \multirow[t]{2}{*}{ Dolor corporal } & $\begin{array}{l}\text { Dolor en alguna } \\
\text { parte del cuerpo } \\
\text { las últimas } \\
4 \text { semanas }\end{array}$ & $3,03 \pm 1,45$ & 14 & 34 & 14 & 11 & 27 & -- \\
\hline & $\begin{array}{l}\text { Interferencia del } \\
\text { dolor para la } \\
\text { realización de } \\
\text { trabajos }\end{array}$ & $2,33 \pm 1,22$ & 30 & 32 & 21 & 9 & 8 & -- \\
\hline \multirow{5}{*}{ Salud general } & $\begin{array}{l}\text { Se pone enferma } \\
\text { más fácil que }\end{array}$ & $3,92 \pm 1,44$ & 15 & 0 & 17 & 14 & 54 & -- \\
\hline & otras personas & & & & & & & \\
\hline & $\begin{array}{l}\text { Esta tan sana } \\
\text { como cualquiera }\end{array}$ & $2,74 \pm 1,52$ & 32 & 19 & 8 & 25 & 16 & -- \\
\hline & $\begin{array}{l}\text { Considera que su } \\
\text { salud empeorara }\end{array}$ & $3,66 \pm 1,38$ & 7 & 20 & 16 & 14 & 43 & -- \\
\hline & $\begin{array}{l}\text { Mi salud es } \\
\text { excelente }\end{array}$ & $2,44 \pm 1,43$ & 40 & 15 & 17 & 17 & 11 & -- \\
\hline
\end{tabular}


tendencia a respuestas de la alternativa 5 (Nunca), mientras que en las pacientes no tratadas las alternativas predominantes fueron las 4 (Solo alguna vez) y 5 (Nunca).

La vitalidad de las pacientes tratadas reveló alternativas de respuestas en el primer y segundo ítems predominantemente hacia la alternativa 1 (Siempre) indicando que siempre se sientes llenas de vitalidad y 2 (Casi siempre) el tercer y cuarto ítems, sus opciones de respuesta estuvieron en la alternativa 4 (Algunas veces) y 5 (Solo alguna vez) señalando que algunas veces o alguna vez se sienten agotados o cansados. El grupo de pacientes no tratadas casi siempre se sienten llenas de vitalidad y tienen mucha energía, así mismo exponen que solo algunas veces se sienten agotados o cansados, en este grupo predominaron las alternativas 2 (Casi siempre) y 4 (Algunas veces).

Los resultados de la evaluación de la salud mental de las pacientes tratadas con TRH demostró que estas pacientes solo alguna vez han estado nerviosas, han tenido el ánimo decaído, se han sentido desanimadas y tristes y afirman que casi siempre se sienten tranquilas y feliz, predominando las alternativas por ítems 5 (Solo alguna vez) y 2 (Casi siempre) mientras que en el grupo de pacientes no tratadas, algunas veces han estado nerviosa o con el ánimo decaído, o desanimado, y por el contrario muchas veces se sienten tranquilas y serenas, y muchas veces se sienten feliz, con un predominio de las alternativas por ítems de 4 (Algunas veces), 3 (Muchas veces) (Tablas IV y V).

La transición de la salud, evaluada a través de los cambios que en esta se producen en el tiempo, evidenció que el grupo de pacientes tratadas afirman que su salud es muy buena comparada con la de hace un año, donde la alternativa predominante fue la 2 (Muy buena) y en el grupo de pacientes no tratadas predomino la alternativa 3 (Buena). (Tablas VI y VII).

El análisis de la calidad vida genérica a través de la suma de los puntajes de cada indicador por sub-dimensión, considerando los valores estándares del SF 36, las cuales indican que de 0 (peor estado de salud) a 100 puntos (mejor estado de salud), deben estar cuantificadas las pacientes de la muestra, evidenciándose en la población estudiada que el grupo que recibió tratamiento totalizó una puntuación de 94,89 mientras que el grupo sin tratamiento obtuvo una puntuación total de 91,82 , la comparación de estos datos permite afirmar que las pacientes tratadas tuvieron un puntaje más cercano a 100 (mejor estado de salud), que las que no recibieron tratamiento (Tabla VIII).

La calidad de vida específica se evaluó a través de la evaluación de los síntomas somáticos, psicológicos y urogenitales de los grupos de pacientes del estudio, en el grupo de menopáusicas no tratadas se observó que sienten molestias moderadas como bochornos, sudoración, calores, molestias al corazón, insomnio, dolor osteomuscular, depresión, irritabilidad, astenia, molestia sexual y vaginal; solo siente molestias leves relacionadas con ansiedad y molestias urinarias. En tanto que el grupo que recibió tratamiento refiere sentir molestias leves relacionadas con bochornos, insomnio, dolor osteomuscular, depresión, irritabilidad, astenia, molestia sexual, urinaria y vaginal y no sienten molestias al corazón ni relacionadas con ansiedad.

Estos resultados permiten establecer que las alternativas predominantes en el grupo de pacientes no tratadas fueron las opciones 1 (Siente molestia leve) y 2 (Siente molestias moderadas) y en el grupo de pacientes tratadas las opciones 0 (No siente molestia) y 1 (Siente molestias leves). La prueba de Mann Whitney fue el estadístico utilizado para establecer el nivel de significancia entre los grupos, donde el nivel de $p<0,05$ fue considerado significativo y el de $p<0,01$ altamente significativo, es decir que la calidad de vida especifica en los grupos evaluados evidencio que el grupo tratado mantiene valores dentro de límites normales a diferencia de los no tratados (Tabla IX).

Se agruparon por indicadores las respectivas sub-dimensiones observándose que los valores encontrados en el grupo de pacientes no tratadas con respecto al grupo de pacientes tratadas en los tres aspectos, somáticos, psicológicos y urogenitales mostraron diferencias significativas entre grupos y al contrastarlos con los valores normales en correspondencia con la población estudiada: Somáticos 4,1 puntos, Psicológicos 4,9 puntos y Urogenitales 1,4 puntos. Se observó que las pacientes tratadas están por debajo de los valores considerados normales en los aspectos somáticos y psicológicos y con predominio de síntomas urogenitales, a diferencia del grupo no tratado en el cual los resultados demuestran que existe una diferencia significativa del otro y de los valores normales en los tres aspectos considerados. Asimismo, el análisis de la calidad de vida específica (Tabla X), evidencia que en el grupo de pacientes no tratadas experimentan síntomas severos, al obtener un puntaje total de 20,79 , mientras que el grupo de pacientes que recibió tratamiento el puntaje total fue de 8,42 , lo que indica que los síntomas experimentados en estas pacientes fueron leves.

En la Tabla XI se presenta el resumen del análisis comparativo de la CV genérica y especifica, medidas mediante los instrumentos SF-36 y MRS 


\section{Tabla IV}

\section{ANÁLISIS DESCRIPTIVO DE LA DIMENSIÓN CALIDAD DE VIDA GENÉRICA: SUB-DIMENSIÓN COMPONENTE MENTAL EN PACIENTES TRATADAS}

\begin{tabular}{|c|c|c|c|c|c|c|c|c|}
\hline \multirow{2}{*}{$\begin{array}{l}\text { Escala } \\
\text { de salud }\end{array}$} & \multirow{2}{*}{$\begin{array}{c}\text { Contenido requerido } \\
\text { del ítems }\end{array}$} & \multirow[t]{2}{*}{$X \pm D E$} & \multicolumn{6}{|c|}{ Distribución porcentual del ítems } \\
\hline & & & 1 & 2 & 3 & 4 & 5 & 6 \\
\hline \multirow{3}{*}{ Rol emocional } & $\begin{array}{l}\text { Tuvo que reducir } \\
\text { el tiempo dedicado } \\
\text { al trabajo por } \\
\text { problemas emocionales }\end{array}$ & $1,85 \pm 0,35$ & 15 & 85 & -- & -- & -- & -- \\
\hline & $\begin{array}{l}\text { Hizo menos de lo que } \\
\text { pudo hacer por } \\
\text { problemas emocionales }\end{array}$ & $1,85 \pm 0,35$ & 15 & 85 & -- & -- & -- & -- \\
\hline & $\begin{array}{l}\text { No hizo su trabajo o } \\
\text { actividades cotidianas por } \\
\text { problemas emocionales }\end{array}$ & $1,85 \pm 0,35$ & 15 & 85 & -- & -- & -- & -- \\
\hline \multirow{2}{*}{ Función social } & $\begin{array}{l}\text { Presencia de } \\
\text { problemas } \\
\text { de salud que } \\
\text { interfieran } \\
\text { con la actividad }\end{array}$ & $4,64 \pm 0,88$ & -- & 7 & 6 & 3 & 84 & -- \\
\hline & $\begin{array}{l}\text { social } \\
\text { Tiempo con } \\
\text { problemas } \\
\text { de salud que } \\
\text { interfieren } \\
\text { con sus actividades } \\
\text { sociales }\end{array}$ & $4,61 \pm 0,88$ & -- & 7 & 6 & 3 & 81 & -- \\
\hline \multirow{4}{*}{ Vitalidad } & $\begin{array}{l}\text { Sentirse lleno } \\
\text { de vitalidad }\end{array}$ & $1,73 \pm 1,02$ & 54 & 30 & 9 & 3 & 4 & -- \\
\hline & $\begin{array}{l}\text { Tener mucha } \\
\text { energía }\end{array}$ & $1,87 \pm 0,98$ & 40 & 44 & 9 & 3 & 4 & -- \\
\hline & Sentirse agotada & $4,54 \pm 0,84$ & -- & 4 & 4 & 33 & 52 & 7 \\
\hline & Sentirse cansada & $4,77 \pm 1,03$ & -- & 4 & 4 & 31 & 33 & 28 \\
\hline \multirow{5}{*}{ Salud mental } & $\begin{array}{l}\text { Ha estado } \\
\text { muy nerviosa }\end{array}$ & $5,24 \pm 1,04$ & -- & -- & 14 & 3 & 28 & 55 \\
\hline & $\begin{array}{l}\text { Sentirse con el } \\
\text { ánimo decaído }\end{array}$ & $5,38 \pm 0,89$ & -- & -- & 8 & 4 & 30 & 58 \\
\hline & $\begin{array}{l}\text { Sentirse tranquila } \\
\text { y serena }\end{array}$ & $1,52 \pm 0,89$ & 68 & 19 & 6 & 7 & -- & -- \\
\hline & $\begin{array}{l}\text { Sentirse desanimada } \\
\text { y triste }\end{array}$ & $5,05 \pm 1,00$ & -- & 4 & -- & 24 & 31 & 41 \\
\hline & Sentirse feliz & $1,64 \pm 0,74$ & 49 & 41 & 7 & 3 & -- & -- \\
\hline
\end{tabular}

respectivamente, efectuado entre ambos grupos. Puede observarse que tanto en la dimensión como en casi todas las sub-dimensiones de la CV genérica no existieron diferencias significativas entre ambos grupos a excepción de la sub-dimensión "Dolor corporal", donde las pacientes tratadas presentaron menores puntuaciones que las no tratadas; diferencias estadísticamente significativa. De igual forma, se realizó con la dimensión CV específica donde si se observaron diferencias altamente significativas entre los dos grupos en todas las sub-dimensiones estudiadas. 
Tabla V

ANÁLISIS DESCRIPTIVO DE LA DIMENSIÓN CALIDAD DE VIDA GENÉRICA: SUB-DIMENSIÓN COMPONENTE MENTAL EN PACIENTES NO TRATADAS

\begin{tabular}{|c|c|c|c|c|c|c|c|c|}
\hline \multirow{2}{*}{$\begin{array}{l}\text { Escala } \\
\text { de salud }\end{array}$} & \multirow{2}{*}{$\begin{array}{c}\text { Contenido requerido } \\
\text { del ítems }\end{array}$} & \multirow[t]{2}{*}{$X \pm D E$} & \multicolumn{6}{|c|}{ Distribución porcentual del ítems } \\
\hline & & & 1 & 2 & 3 & 4 & 5 & 6 \\
\hline \multirow{3}{*}{ Rol emocional } & $\begin{array}{l}\text { Tuvo que reducir } \\
\text { el tiempo dedicado } \\
\text { al trabajo por problemas } \\
\text { emocionales }\end{array}$ & $1,57 \pm 0,49$ & 43 & 57 & -- & -- & -- & -- \\
\hline & $\begin{array}{l}\text { Hizo menos de lo que } \\
\text { pudo hacer por } \\
\text { problemas emocionales }\end{array}$ & $1,57 \pm 0,49$ & 43 & 57 & -- & -- & -- & -- \\
\hline & $\begin{array}{l}\text { No hizo su trabajo } \\
\text { o actividades cotidianas } \\
\text { por problemas emocionales }\end{array}$ & $1,62 \pm 0,48$ & 38 & 62 & -- & -- & -- & -- \\
\hline \multirow[t]{2}{*}{ Función social } & $\begin{array}{l}\text { Presencia de } \\
\text { problemas de salud } \\
\text { que interfieran } \\
\text { con la actividad } \\
\text { social }\end{array}$ & $3,9 \pm 1,25$ & 7 & 10 & 11 & 30 & 42 & -- \\
\hline & $\begin{array}{l}\text { Tiempo con } \\
\text { problemas } \\
\text { de salud que } \\
\text { interfieren } \\
\text { con sus actividades } \\
\text { sociales }\end{array}$ & $3,8 \pm 1,2$ & 7 & 10 & 11 & 33 & 39 & -- \\
\hline \multirow{4}{*}{ Vitalidad } & $\begin{array}{l}\text { Sentirse llena } \\
\text { de vitalidad }\end{array}$ & $2,17 \pm 1,36$ & 44 & 28 & 3 & 17 & 8 & -- \\
\hline & $\begin{array}{l}\text { Tener mucha } \\
\text { energía }\end{array}$ & $2,4 \pm 1,50$ & 39 & 25 & 6 & 22 & 3 & 5 \\
\hline & Sentirse agotada & $4,04 \pm 1,42$ & 8 & 11 & 5 & 34 & 29 & 13 \\
\hline & Sentirse cansada & $4,0 \pm 1,40$ & 8 & 11 & 5 & 37 & 27 & 12 \\
\hline \multirow{5}{*}{ Salud mental } & $\begin{array}{l}\text { Ha estado muy } \\
\text { nerviosa }\end{array}$ & $4,39 \pm 1,51$ & 10 & -- & 9 & 35 & 14 & 32 \\
\hline & $\begin{array}{l}\text { Sentirse con el } \\
\text { ánimo decaído }\end{array}$ & $4,42 \pm 1,82$ & 17 & 3 & -- & 22 & 17 & 41 \\
\hline & $\begin{array}{l}\text { Sentirse tranquila } \\
\text { y serena }\end{array}$ & $3,09 \pm 1,7$ & 26 & 16 & 15 & 20 & 12 & 11 \\
\hline & $\begin{array}{l}\text { Sentirse desanimada } \\
\text { y triste }\end{array}$ & $3,97 \pm 1,7$ & 14 & 13 & 8 & 22 & 13 & 30 \\
\hline & Sentirse feliz & $2,67 \pm 1,66$ & 45 & 4 & 6 & 33 & 8 & 4 \\
\hline
\end{tabular}


Tabla VI

ANÁLISIS DESCRIPTIVO DE LA DIMENSIÓN CALIDAD DE VIDA GENÉRICA: SUB-DIMENSIÓN TRANSICIÓN DE SALUD EN PACIENTES TRATADAS

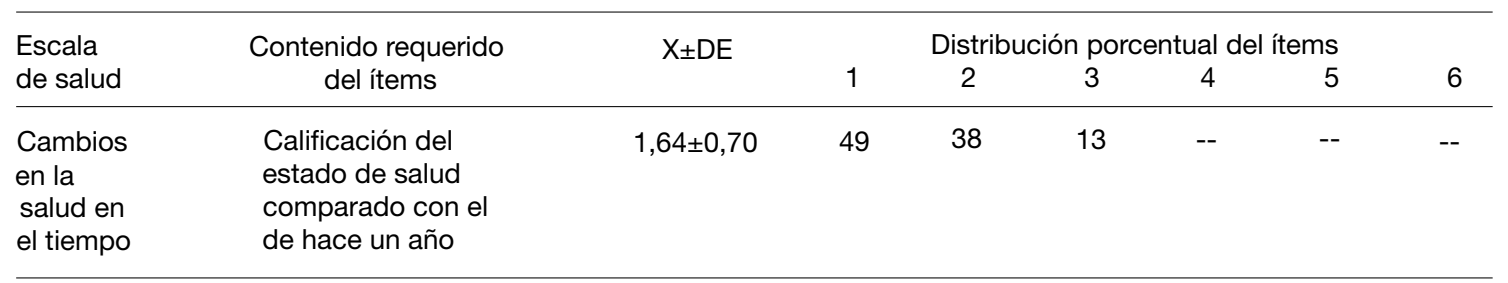

Tabla VII

ANÁLISIS DESCRIPTIVO DE LA DIMENSIÓN CALIDAD DE VIDA GENÉRICA: SUB-DIMENSIÓN TRANSICIÓN DE SALUD EN PACIENTES NO TRATADAS

\begin{tabular}{|c|c|c|c|c|c|c|c|c|}
\hline \multirow{2}{*}{$\begin{array}{l}\text { Escala } \\
\text { de salud }\end{array}$} & \multirow{2}{*}{$\begin{array}{c}\text { Contenido requerido } \\
\text { del ítems }\end{array}$} & \multirow[t]{2}{*}{$\mathrm{X} \pm \mathrm{DE}$} & \multicolumn{6}{|c|}{ Distribución porcentual del ítems } \\
\hline & & & 1 & 2 & 3 & 4 & 5 & 6 \\
\hline $\begin{array}{l}\text { Cambios en } \\
\text { la salud en } \\
\text { el tiempo }\end{array}$ & $\begin{array}{l}\text { Calificación del } \\
\text { estado de salud } \\
\text { comparado con el } \\
\text { de hace un año }\end{array}$ & $2,95 \pm 1,19$ & 17 & 15 & 31 & 30 & 7 & -- \\
\hline
\end{tabular}

Tabla VIII

\section{ANÁLISIS DESCRIPTIVO DE LA CALIDAD DE VIDA GENÉRICA}

\begin{tabular}{lcc}
\hline $\begin{array}{l}\text { Análisis/ } \\
\text { Alternativa }\end{array}$ & $\begin{array}{c}\text { Pacientes no } \\
\text { tratadas } \\
\text { (puntuación) }\end{array}$ & $\begin{array}{c}\text { Pacientes } \\
\text { tratadas } \\
\text { (puntuación) }\end{array}$ \\
\hline Función física & 24,02 & 24,46 \\
Rol físico & 6,07 & 7,36 \\
Dolor corporal & 5,36 & $3,21^{*}$ \\
Salud general & 12,76 & 13,37 \\
Rol emocional & 4,76 & 5,5 \\
Función social & 7,7 & 9,25 \\
Vitalidad & 12,61 & 12,91 \\
Salud mental & 18,54 & 18,83 \\
Componente físico & 48,21 & 48,40 \\
Componente mental & 43,61 & 46,49 \\
Puntuación total & 91,82 & 94,89 \\
\hline
\end{tabular}

${ }^{*} \mathrm{p}<0,05$ Prueba de Mann Whitney entre grupos de pacientes no tratadas y tratadas.
Tabla IX

ANÁLISIS DESCRIPTIVO DE LA DIMENSIÓN CALIDAD DE VIDA ESPECÍFICA: SÍNTOMAS SOMÁTICOS, SÍNTOMAS PSICOLÓGICOS Y SÍNTOMAS UROGENITALES

\begin{tabular}{lcc}
\hline Síntomas & $\begin{array}{c}\text { Pacientes } \\
\text { no tratadas } \\
\mathrm{X} \pm \mathrm{DE}\end{array}$ & $\begin{array}{c}\text { Pacientes } \\
\text { tratadas } \\
\mathrm{X} \pm \mathrm{DE}\end{array}$ \\
\hline Bochornos & $2,05 \pm 1,42$ & $0,69 \pm 0,93^{\star *}$ \\
Palpitaciones & $1,64 \pm 1,02$ & $0,44 \pm 0,70^{\star *}$ \\
Insomnio & $2,34 \pm 1,58$ & $0,85 \pm 1,33^{\star *}$ \\
Dolor osteomuscular & $2,13 \pm 1,26$ & $0,86 \pm 1,08^{\star *}$ \\
Depresión & $1,67 \pm 1,32$ & $0,53 \pm 1,07^{\star *}$ \\
Irritabilidad & $1,90 \pm 1,45$ & $0,62 \pm 0,92^{\star *}$ \\
Ansiedad & $1,25 \pm 1,29$ & $0,35 \pm 0,79^{\star *}$ \\
Astenia & $1,85 \pm 1,27$ & $0,81 \pm 0,90^{\star *}$ \\
Molestia sexual & $2,24 \pm 1,32$ & $1,21 \pm 1,72^{\star}$ \\
Molestia urinaria & $1,47 \pm 1,42$ & $1,08 \pm 1,48^{\star}$ \\
Molestia vaginal & $2,24 \pm 1,42$ & $0,98 \pm 1,32^{\star *}$ \\
Síntomas somáticos $\$$ & $8,17 \pm 5,28$ & $2,34 \pm 4,04^{\star *}$ \\
Síntomas psicológicos \& & $6,67 \pm 5,33$ & $2,31 \pm 3,68^{\star *}$ \\
Síntomas urogenitales -- & $5,95 \pm 4,16$ & $3,27 \pm 4,52^{\star *}$ \\
\hline
\end{tabular}

\$ Suma de los ítems 1, 2, 3 y 4.

\& Suma de los ítems $5,6,7$ y 8.

-- Suma de los ítems 9, 10 y 11.

${ }^{*} p<0,05$ Prueba de Mann Whitney entre grupos de pacientes no tratadas y tratadas.

${ }^{* *} p<0,01$ Prueba de Mann Whitney entre grupos de pacientes no tratadas y tratadas. 
Tabla X

ANÁLISIS DESCRIPTIVO DE LA CALIDAD DE VIDA ESPECÍFICA

\begin{tabular}{lcccc}
\hline Intensidad/Síntomas & $\begin{array}{c}\text { Porcentaje población } \\
\text { latinoamericana* } \\
\mathrm{X} \pm \mathrm{DE}\end{array}$ & $\begin{array}{c}\text { Pacientes tratadas } \\
\mathrm{y} \text { no tratadas } \\
\mathrm{X}(\mathrm{n}=200)\end{array}$ & $\begin{array}{c}\text { Pacientes } \\
\text { no tratadas } \\
\mathrm{X}(\mathrm{n}=100)\end{array}$ & $\begin{array}{c}\text { Pacientes } \\
\text { tratadas } \\
\mathrm{X}(\mathrm{n}=100)\end{array}$ \\
\hline Puntuación total & $10,4 \pm 8,8$ & 14,60 & 20,79 & 8,42 \\
Síntomas somáticos & $4,9 \pm 4,5$ & 5,25 & 8,17 & 2,34 \\
Síntomas psicológicos & $4,1 \pm 3,6$ & 4,49 & 6,67 & 2,31 \\
Síntomas urogenitales & $1,4 \pm 2,2$ & 4,61 & 5,95 & 3,27 \\
\hline
\end{tabular}

Escasa o Nula (0 - 4), Leve (5 - 8), Moderado (9 - 15), Severo ( >16 ) puntos.

* Datos procedentes de la estandarización de la Escala MRS (Heinemann y cols, 2004)

Tabla XI

\section{ANÁLISIS COMPARATIVO DE LA CALIDAD DE VIDA GENÉRICA Y ESPECÍFICA POR EL SF 36 Y MRS}

\begin{tabular}{|c|c|c|}
\hline $\begin{array}{l}\text { Intensidad/ } \\
\text { Síntomas }\end{array}$ & $\begin{array}{l}\text { Pacientes } \\
\text { no tratadas } \\
\text { (Puntuación) }\end{array}$ & $\begin{array}{c}\text { Pacientes } \square \\
\text { tratadas } \bigotimes \\
\text { (Puntuación) }\end{array}$ \\
\hline \multicolumn{3}{|l|}{ SF-36 } \\
\hline Puntuación total & 91,82 & 94,89 \\
\hline Componente físico & 48,21 & 48,40 \\
\hline Componente mental & 43,61 & 46,49 \\
\hline Función física & 24,02 & 24,46 \\
\hline Rol físico & 6,07 & 7,36 \\
\hline Dolor corporal & 5,36 & $3,21^{*}$ \\
\hline Salud general & 12,76 & 13,37 \\
\hline Rol emocional & 4,76 & 5,5 \\
\hline Función social & 7,7 & 9,25 \\
\hline Vitalidad & 12,61 & 12,91 \\
\hline 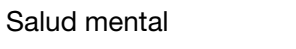 & 18,54 & 18,83 \\
\hline \multicolumn{3}{|l|}{ MRS区 } \\
\hline Puntuación total & 20,79 & 8,42 \\
\hline Síntomas somáticos & 8,17 & $2,34^{\star \star}$ \\
\hline Síntomas psicológicos & 6,67 & $2,31^{\star *}$ \\
\hline Síntomas urogenitales & 5,95 & $3,27^{\star \star}$ \\
\hline
\end{tabular}

${ }^{*} \mathrm{p}<0,05$ Prueba de Mann Whitney entre grupos de pacientes no tratadas y tratadas.

${ }^{\star *} \mathrm{p}<0,01$ Prueba de Mann Whitney entre grupos de pacientes no tratadas y tratadas.

\section{DISCUSIÓN}

Para el año 2030, se estima que 47 millones de mujeres cada año alcanzaran la menopausia. Aproximadamente un $75-80 \%$ de las mujeres experimentan síntomas menopáusicos, aunque la mitad aproximadamente encuentra los síntomas como molestos, sólo el $20-30 \%$ tienen síntomas severos (27). Es por ello que en los últimos años, son cada vez más frecuentes los estudios que incorporan las escalas de la calidad de vida, como instrumento para valorar la acción de una intervención farmacológica como la $\mathrm{TRH}$, bien sea con tibolona, estrógenos equinos, o raloxifeno (10).

La edad de la menopausia, entre 45 y 55 años, no ha cambiado, pero sí que se ha modificado la esperanza de vida de la mujer; por tanto cada vez más existirá un mayor número de mujeres posmenopáusicas. Los resultados obtenidos permitieron determinar una edad promedio de la aparición de la menopausia en ambos grupos de 48 años, cercano al promedio de 49,1 años reportada por un estudio multicéntrico latinoamericano (28), aunque superior a la encontrada en otra investigación venezolana donde se reportó una edad de 45,8 años. Asimismo, en cuanto al tipo de menopausia los resultados obtenidos de esta población también difieren de los encontrados por estos mismos autores; quienes reportaron una frecuencia de menopausia natural del $69,2 \%$, mientras que en este grupo predominó la menopausia quirúrgica (48\%) (13).

$\mathrm{La}$ TRH no debe ser un tratamiento obligado en la menopausia; su objetivo principal es mejorar la calidad de vida y prevenir determinadas patologías (28). No obstante, a la luz de los conocimientos 
actuales y a pesar de las controversias surgidas a partir de la publicación del estudio WHI, la TRH sigue siendo la medida más efectiva para los síntomas menopáusicos y la recomendación es que después de cinco años se debe realizar una evaluación individual y así decidir sobre la conveniencia y la seguridad de continuar con tal tratamiento (29). En tal sentido, en el grupo de mujeres tratadas el tiempo de uso del tratamiento hormonal fue menor a 5 años (3,2 años).

Esta investigación reveló que fármacos antiandrógenos como la tibolona, eran la principal terapéutica empleada en las mujeres que recibían tratamiento, con una frecuencia del 32\%; seguida de los estrógenos equinos conjugados y los estrógenos transdérmicos. Estos resultados difieren de los encontrados en otra población venezolana por Álvarez y cols (13), donde la mayoría de las pacientes $(80 \%)$ eran tratadas con combinaciones de estrógenos más progestágenos; esta diferencia encontrada puede estar asociada con el gran número de mujeres histerectomizadas presente en el grupo de menopáusicas tratadas, lo cual excluye el riesgo de desarrollar malignidades en el endometrio a consecuencia del uso de la TRH.

Aunque el instrumento SF-36 es una de las herramientas mayormente empleadas para determinar la $\mathrm{CV}$ y ha sido utilizados en numerosos estudios dedicado a la salud de las mujeres, son pocas las investigaciones realizadas en menopáusicas donde se haya medido la CV general por medio este instrumento, por lo cual resulto novedoso e interesante su utilización en esta investigación. Hartmann y cols (30), utilizando este instrumento encontraron que en menopáusicas quirúrgicas que presentaban, dolor o depresión antes de la histerectomía, al compararlas con aquellas que no presentaron ni dolor ni depresión, presentaban un riesgo de 3 a 5 veces más de presentar una disminución de su $\mathrm{CV}$.

Un estudio mexicano, en el cual se empleo otro tipo de cuestionario genérico (Coop/Wonka), no encontró diferencias significativas en las dimensiones de forma física, sentimientos, actividades sociales y percepción del dolor entre climatéricas con o sin $\mathrm{TRH}$; más no así en el estado de salud que fue significativamente superior en el grupo de mujeres tratadas (31). Otro instrumento genérico empleado para el análisis de la CV, es el instrumento Europeo de Calidad de Vida (EuroQol); las investigaciones realizadas por Welton y cols (32) y Veerus y cols (33), utilizando esta escala determinaron que después de utilizar combinaciones de estrógenos equinos conjugados mas progestágenos como $\mathrm{TRH}$, se encontraban mejoras significativas en los indicadores de síntomas vasomotores, problemas del sueño y función sexual; más no así en la calidad de vida general observada.

En el presente estudio la función física se observó mas afectadas en el grupos de mujeres no tratadas, aunque no resulto significativo, mientras que el dolor corporal si mostró diferencias entre los dos grupos, siendo mayor la intensidad en las mujeres no tratadas. Esto denota que las menopáusicas que no reciben tratamiento presentan dolor corporal en alguna parte del cuerpo y este ocasiona interferencias para la realización de trabajos. En forma parcial, los resultados de este estudio concuerdan con los obtenidos con Hays y cols (17), quienes encontraron pequeñas diferencias, aunque estadísticamente significativas, sólo para las dimensiones de función física, dolor y alteraciones del sueño.

En cuanto al componente mental evaluado por este instrumento genérico no se encontraron diferencias significativas en cuanto al rol emocional, vitalidad, salud mental, o función social. Estos resultados tienen similitud con los reportados por Yaffe y cols (34), quienes luego de dos años de seguimiento en mujeres posmenopáusicas que recibían dosis ultra bajas de estrógenos transdérmicos no encontraron diferencias significativas en ninguno de los 36 ítems de esta escala; aunque esto no constituye una evidencia consistente ya que estos resultados dependen de los niveles basales de estrógenos endógenos.

Asimismo, Hartman y cols (35), evaluaron mediante este instrumento la relación entre el dolor y la calidad de vida en climatéricas con depresión mayor, encontrando que la intensidad del dolor se relacionaba con la severidad de la depresión y que la CV disminuía en las climatéricas con depresión, reflejada en las bajas puntuaciones de las escalas de rol emocional y función social.

De igual forma, pudo observarse que en cuanto a la puntuación total obtenidas en la medición total de la CV genérica no hubo diferencias estadísticamente significativas entre ambos grupos, resultados que concuerdan con los de las investigaciones efectuadas por Texón y Márquez (31), Welton y cols (32), Veerus y cols (33) y Hays y cols (17).

Los resultados de la aplicación del instrumento de CV especifico, el MRS, revelaron una diferencia altamente significativa entre los dos grupos, presentando las mujeres tratadas mejores puntuaciones en las tres sub-dimensiones evaluadas, por debajo tanto de los valores de referencia para poblaciones latinas (27) como de los encontrados para la población venezolana y latinoamericana en el estudio de Chedraui y cols (28), salvo para la sintomatología urogenital al compararla con ambos estudios. Estos resultados son concordantes 
con los reportados por Dinger y cols (36), quienes pudieron demostrar que la TRH estaba relacionada con una mejoría de alrededor del $30 \%$, tanto en la puntuación total de la escala como en la puntuación de los tres dominios evaluados; asimismo encontró una similar relativa mejora después de la terapia hormonal al estratificar la sintomatología según los grados de severidad.

De igual manera, Chedraui y cols (28), refieren que más de la mitad de la población latinoamericana presentan puntuaciones de moderadas a severas al aplicarles esta escala, resultado que concuerda con las puntuaciones obtenidas en el grupo de mujeres no tratadas. Asimismo, estos autores encontraron que el uso de la TRH se asociaba con un menor riesgo de alteración en la CV $(\mathrm{OR}: 0,65)$, contrario a esto, el uso de alternativas para el manejo de la menopausia se relacionaba con síntomas más severos de la menopausia.

Asimismo, pudo establecerse que en el grupo de las mujeres tratadas la sintomatología reportada era leve, mientras que en las que no recibían la $\mathrm{TRH}$ era moderada. En este sentido, Heinemann y cols (24) reportaron que con el tratamiento hormonal se obtiene una mejoría tanto en todos los componentes de la escala como en la intensidad de la sintomatología, con un promedio del $36 \%$ en la puntuación total, por lo que este instrumento pudiese tener un valor predictivo para evaluar el tratamiento médico.

Respecto a las funciones cognitivas, medida en este instrumento como astenia, se encontró una diferencia altamente significativa $(p<0,01)$ en el grupo de las menopáusicas tratadas, sin embargo, este resultado difiere del reportado por Maki y cols (37), quienes no encontraron diferencias significativas ni en la cognición ni en la calidad de vida en mujeres con y $\sin \mathrm{TRH}$, evaluadas mediante dos instrumentos de CV: "Greene Climacteric Scale" y "Utian Quality of Life Scale". Sólo se observo mejoría en lo relacionado a los síntomas vasomotores.

Resulta interesante resaltar que los puntajes de los dominios somáticos y psicológicos son mayores respecto al dominio urogenital, a pesar de existir una diferencia altamente significativa en cuanto a la sintomatología urogenital en las menopáusicas bajo TRH al compararse con el grupo de las no tratadas. Esta misma observación, de que habría un mayor compromiso en la esfera psicológica y somática que en la urogenital ha sido descrita en otros países latinoamericanos como Chile, a diferencia de lo que se ha observado en países de otros continentes (23).

Otro hallazgo importante de esta investigación fueron las marcadas diferencias encontradas en el grupo de pacientes no tratadas con respecto al grupo de pacientes bajo TRH en los tres aspectos medidos por la escala MRS; los cuales al contrastarlos con los valores para la población latinoamericana obtenidos de la estandarización del instrumento, se observó que las pacientes tratadas están por debajo de los valores considerados normales en los aspectos somáticos y psicológicos, más no así respecto a los de síntomas urogenitales donde la puntuación obtenida es mayor.

Sin embargo, el promedio de las puntuaciones obtenidas al unir ambos grupos es similar a la reportada por Chedraui y cols (28), tanto para la población latinoamericana como para la población venezolana, en los promedios de la puntuación general y de los síntomas somáticos y psicológicos, observándose nuevamente mayor afectación respecto a la sintomatología urogenital.

No debe olvidarse que el concepto de CV abarca diferentes dimensiones y puede ser influidos por factores culturales, sociales y económicos. Monterrosa y cols (38), estudiaron la CV específica en mujeres colombianas menopáusicas de tres grupos étnicos diferentes utilizando esta escala, encontrando que las mujeres hispánicas tenían mejor $\mathrm{CV}$ que las afrodescendientes y las indígenas; lo cual pudiese estar influenciado por las diferencias socioeconómicas entre estos grupos poblacionales. En este sentido, las mujeres negras presentaban mayor deterioro psicológico y más síntomas somáticos, mientras que las mujeres indígenas presentaban más síntomas urogenitales.

El rol de los profesionales de salud debería también enfocarse en la orientación, educación y apoyo emocional de las mujeres; lamentablemente, muchos profesionales basan su atención en un modelo "tradicional" enfocado sólo a la patología que presenta la mujer, sin evaluarla integralmente (39). En la medida que la atención de salud se focalice en las necesidades de las mujeres menopáusicas se podrá lograr que ellas sean activas en su propio cuidado.

No debe olvidarse que los cuestionarios de CV no deben sustituir a las evaluaciones sintomáticas, analíticas o morfológicas, sino por el contrario ser un complemento que permita introducir algo tan trascendente como la visión del propio paciente sobre su percepción de salud (19). Estos resultados demuestran la capacidad de discriminación y predicción de los instrumentos de CV específica, por lo que son útiles para la realización de ensayos clínicos y monitorizar los cambios en salud que un tratamiento específico, como la $\mathrm{TRH}$, puede producir sobre una persona (40).

Los resultados obtenidos permitieron encontrar 
mayores diferencias entre los grupos con el uso de un instrumento específico como la escala MRS, coincidiendo con lo formulado tanto por Tuesca (18) como por Velarde y Ávila (41); asimismo este tipo de instrumentos son mas sensibles para detectar mejoras en la salud (42). A pesar de esto, no debe descartarse el uso de cuestionario genérico ya que estos permiten discriminar y comparar entre diferentes enfermedades. Además debe recordarse lo expuesto por Aranibar (40), fiel defensor de esta clase de instrumentos, ya que éstos permiten detectar efectos colaterales o imprevistos de los tratamientos médicos y realizar comparaciones con la población en general.

La medición de CV debe ser un parámetro incluido en la práctica clínica, ya que permite valorar las intervenciones terapéuticas, así como también los posibles efectos adversos a corto y largo plazo, con respecto al proceso que viven estas pacientes. Esta situación será posible en la medida que le logre entender el concepto de CV en su real dimensión y pueda ligarse al significado de lo que comúnmente se llama en la práctica clínica una "atención integral". Es importante considerar que las mujeres que reciben una mejor atención al momento de enfrentarse al diagnóstico, con un equipo profesional que resuelve sus dudas e informa de acuerdo a sus inquietudes, demuestran sentirse más seguras y apoyadas en esta experiencia, lo que revela mejores índices de $\mathrm{CV}$ en relación a las pacientes que no reciben este tipo de atención (39).

Estos hallazgos encontrados en los 2 grupos evaluados en este trabajo son un aporte al conocimiento de la CV en relación a la percepción de salud de las mujeres menopáusicas de la población estudiada. Es indudable que aún falta desarrollar otros estudios que permitan evaluar otros componentes de la calidad de vida relacionadas con la salud como por ejemplo variables psicológicas, sociales, económicas, patológicas e intervenciones terapéuticas

Si bien el tipo y diseño de la investigación, comparativo y transeccional, presenta algunas limitaciones para efectuar un análisis de causalidad directa, los resultados del trabajo informa el beneficio en la CVRS de mujeres posmenopáusicas sometidas a $\mathrm{TRH}$. Por otra parte, tradicionalmente en Venezuela la evaluación de las consecuencias que la menopausia tiene sobre la mujer se ha hecho a partir del análisis de la sintomatología clínica o los conocimientos sobre el climaterio y menopausia como puede verse en la investigación de Álvarez y cols (13), las cuales han pretendido proporcionar información indirecta para la evaluación de la eficacia de las intervenciones médicas.
Esta investigación viene a ser de los primeros trabajos venezolanos en considerar tanto los fenómenos que ocurren en las esferas física, psicológica y urogenital de la mujer, como su propia percepción sobre los cambios que experimentan durante esta etapa de sus vidas. Puesto que si bien el trabajo de Chedraui y cols (28), incluyó la población de Venezuela, este es un estudio multicéntrico efectuado en 12 países latinoamericanos liderizado por investigadores de otras naciones.

Otra limitación que se puede mencionar es no haber discriminado en relación a algunos aspectos tanto de índole sociodemográficos como la etnia; que es otro asunto a tener en cuenta cuando se comparan poblaciones en términos de severidad de los síntomas menopáusicos (38). Asimismo, tampoco fueron considerados aspectos como la obesidad, medida mediante el índice de masa corporal, o factores relacionados con la pareja como la disfunción eréctil o la eyaculación precoz, los cuales han estados vinculados con la severidad se la sintomatología menopáusica (28).

\section{CONCLUSIÓN}

Las pacientes bajo TRH perciben una mayor CV específica respecto a las pacientes menopáusicas que no la reciben, presentando síntomas somáticos o psicológicos menos severos. Los síntomas de la esfera urogenital son percibidos con mayor intensidad que lo considerado normal para la población latinoamericana. La escala MRS, instrumento específico de CV utilizado en esta investigación, mostró ser útil para demostrar las diferencias en la percepción de salud al recibir una intervención farmacológica como la TRH.

Es necesario incluir en un protocolo de investigación a las pacientes climatéricas que actualmente se encuentren bajo terapia hormonal de reemplazo, y efectuar un seguimiento a mediano y largo plazo para identificar con oportunidad los beneficios inherentes al tratamiento de sustitución.

\section{BIBLIOGRAFÍA}

1. Sennot L. La mujer de edad avanzada en las Américas. Problemas y posibilidades. En: Gómez, E., (Ed.) Género, mujer y salud en las Américas. Washington, DC: OPS; 2003. pp. 114-123.

2. Organización Mundial de la Salud. The World Organization quality of life instruments (sf). [Documento en línea] Disponible en: http://www.who.int/evidence/ assessmentinstruments/qol/ql1.htm. Fecha de recuperación: 27 de septiembre de 2008.

3. Ramones I. Calidad de vida relacionada a salud e hipertensión arterial. Boletín Médico de Postgrado 2004; 20 (4):13-16. 
4. Roizen M, Figueroa C, Salvia L, Bauer G, Bevilacqua $\mathrm{S}$, Bordato $\mathrm{A}$, et al. Calidad de vida relacionada con la salud en niños con enfermedades crónicas: comparación de la visión de los niños, sus padres y sus médicos. Archivos Argentinos de Pediatría 2007;105(4):305-13.

5. Montes ML. Calidad de vida. Revista Mexicana de Medicina Física y Rehabilitación 2006;18:5-6.

6. Leno D, Lozano M, Leno J. Estereotipo psico-sociocultural de la menopausia en mujeres rurales. Revista electrónica de Enfermería Global 2006;(9). [Periódico en línea]. Disponible en: http://www.um.es/eglobal/. Fecha de recuperación: 29 de julio de 2008.

7.Chacín B. Citología endometrial en pacientes posmenopáusicas con terapia hormonal. Rev Obstet Ginecol Venezolana 2008;68(2):73-80.

8. López A, González R. Actualización en menopausia: abordaje desde atención primaria. Revista Valenciana de Medicina de Familia. 2008;15:4-9.

9. Yanes M, Chio I. Calidad de vida en la mujer de edad mediana. Revista Cubana de Medicina General Integral 2004;2(20). [Periódico en línea]. Disponible en: http://scielo.sld.cu/scielo.php?script=sci_arttext\&pid= S086421252004000200003\&lng=es\&nrm=iso. Fecha de recuperación: 24 de junio de 2008.

10. Juliá M, Romeu A, García Y. Estudio piloto para valorar los cambios en la calidad de vida en mujeres postmenopáusicas sintomáticas tras la administración de cimicifuga racemosa L. Evaluada con la escala Cervantes. Revista Iberoamericana de Fertilidad 2006; 23(3):193-201.

11. Triviño Z, Stiepovich J, Merino J. Factores predictores de conductas promotoras de salud en mujeres peripost-menopáusicas de Cali, Colombia. Colombia Médica 2007;38(4):395-407.

12. Ortiz, N, Mariño C, Méndez B, Olarte N, Blumel JE. Deterioro de la calidad de vida durante el climaterio. Revista Colombiana de Menopausia 2001;7(2):20110.

13. Álvarez R, Martín E, Bordones M. Conocimiento y actitud sobre el climaterio en mujeres entre 40 y 50 años. Revista de Obstetricia y Ginecología Venezolana 2008;68(1):32-40.

14. Rohde A. Psychological aspects of the menopause. Annals of General Psychiatry 2008; 7(Suppl1):S72 doi:10.1186/1744-859X-7-S1-S72 [Periódico en línea] Disponible en: http://www.biomedcentral.com/content/ files/pdf/1744-859X-7.

15. Escobar $F$, Chica $H$, Cuevas $F$. Trastornos del sueño relacionados con el climaterio femenino y su tratamiento. Revista Colombiana de Obstetricia y Ginecología 2008;59(2):131-9.

16. Anderson G, Limacher M. Effects of Conjugated Equine Estrogen in Postmenopausal women with hysterectomy. The Women's Health Initiative Randomized Controlled Trial. JAMA 2004;291(14):1701-12.

17. Hays J, Ockene J, Brunner R, Kotchen J, Manson $\mathrm{J}$, Patterson R, et al. Effects of estrogen plus progestin on health-related quality of life. $\mathrm{N}$ Engl $\mathrm{J}$ Med 2003;348(19):1839-54

18. Tuesca R. La calidad de vida, su importancia y cómo medirla. Salud Uninorte 2005;21(2):76-86.
19. Monés J. ¿Se puede medir la calidad de vida? ¿Cuál es su importancia? Cirugía Española 2004;76(2):71-7.

20. Vilagut G, Ferrer M, Rajmil L, Rebollo P, PermanyerMiralda G, Quintana JM, et al. El Cuestionario de salud SF-36 español: una década de experiencia y nuevos desarrollos. Revista Gaceta Sanitaria 2005;19(2):13550.

21. Lugo LH, García HI, Gómez Carlos. Confiabilidad del cuestionario de calidad de vida en salud SF-36 en Medellín, Colombia. Revista de la Facultad Nacional de Salud Pública 2006;24(2):37-50.

22. Síseles NO, Pecci C, Gutiérrez AP, Mir-Candal LP. Impacto de la menopausia sobre calidad y expectativa de vida de la mujer en la mediana edad. En: Síseles, N. (Ed.). Información y formación para el manejo actual de la mujer en su climaterio. Buenos Aires, Argentina: Editorial Ascune Hermanos; 2005. 574 pp.

23. Aedo S, Irribarra C, Porcile A. Calidad de vida relacionada con el climaterio en una población chilena de mujeres saludables. Revista Chilena de Obstetricia y Ginecología 2006;71(6):402-9.

24. Heinemann K, Ruebig A, Potthoff $P$, Schneider HP, Strelow F, Heinemann LAJ, Do MT. The Menopause Rating Scale(MRS) scale: a methodological review. Health Qual Life Outcomes 2004;2:45. doi:10.1186/1477-7525-2-45. [Periódico en línea] Disponible en: http://www.hqlo.com/content/2/1/45. Fecha de recuperación: 16 de julio de 2008.

25. Heinemann LAJ, Do MT, Strelow F, Gerbsch S, Schnitker J, Schneider HPG. The Menopause Rating Scale (MRS) as outcome measure for hormone treatment? A validation study. Health and Quality of Life Outcomes 2004;2:67 doi:10.1186/1477-7525-267. [Periódico en línea] Disponible en: http://www. hqlo.com/content/2/1/67. Fecha de recuperación: 16 de julio de 2008.

26. Heinemann LAJ, Potthoff P, Schneider HPG. International versions of the Menopause Rating Scale (MRS). Health and Quality of Life Outcomes 2003; 1:28. [Periódico en línea] Disponible en: http://www.hqlo.com/ content/1/1/28. Fecha de recuperación: 16 de julio de 2008.

27. Palacios S. Advances in hormone replacement therapy: making the menopause manageable. BMC Women's Health 2008;8:22. [Periódico en línea]. Disponible en: http://www.biomedcentral.com/14726874/8/22. Fecha de recuperación: 7 de febrero de 2009 .

28. Chedraui $P$, Blümel JE, Barón G, Belzares E, Bencosme A, Calle A, Danckers L, et al. Calidad de vida alterada entre mujeres de edad media: un estudio multicéntrico latinoamericano. Revista Colombiana de Menopausia 2009;15(1):42-53.

29. Zárate A, Hernández M, Basurto L. Estado actual del tratamiento hormonal en la menopausia. Revista de la Facultad de Medicina de la Universidad Autónoma de México 2005;48(1):14-7.

30. Hartmann KE, Ma C, Lamvu GM, Langenberg PW, Steege JF, Kjerulff KH. Quality of life and sexual function after hysterectomy in women with preoperative pain and depression. Obstet Gynecol 2004;104(4):701-9. 
31. Téxon O, Márquez F. Calidad de vida en mujeres climatéricas con y sin terapia hormonal de reemplazo. Revista Médica del Instituto Mexicano de los Seguros Sociales 2006;44(6):541-45.

32. Welton A, Vickers M, Kim J, Ford D, Lawton B, MacLennan $A$, et al. Health related quality of life after combined hormone replacement therapy: randomised controlled trial. BMJ 2008;337:a1190. [Periódico en línea]. Disponible en: http://bmj.com/cgi/content/ full/337/aug21_2/a1190\#BIBL. Fecha de recuperación: 25 de agosto de 2008.

33. Veerus P, Fischer K, Hovi S, Karro H, Rahu M, Hemminki $\mathrm{E}$. symptom reporting and quality of life in the estonian postmenopausal Hormone Therapy Trial. BMC Women's Health 2008;8:5 [Periódico en línea] Disponible en: http://www.biomedcentral.com/14726874/8/5. Fecha de recuperación: 19 de agosto de 2008.

34. Yaffe K, Vittinghoff E, Ensrud KE, Johnson KC, Diem S, Hanes V, Grady D. Effects of ultra-low-dose transdermal estradio on cognition and health-related quality of life. Archives of Neurology 2006;63(7):945-50.

35. Hartman JM, Berger A, Baker K, Bolle J, Handel D, Mannes A, et al. Quality of life and pain in premenopausal women with major depressive disorder: The POWER Study. Health Qual Life Outcomes 2006; 4:2. doi: 10.1186/1477-7525-4-2. [Periódico en línea] Disponible en: http://www.ncbi.nlm.nih.gov/pmc/articles/ PMC1373611/. Fecha de recuperación: 8 de mayo de 2008.

36. Dinger J, Zimmermann T, Heinemann LAJ, Stoehr D.
Quality of life and hormone use: new validation results of MRS scale. Health and Quality of Life Outcomes 2006;4(32):1-5. doi:10.1186/1477-7525-4-32. [Periódico en línea] Disponible en: http://www.hqlo.com/ content/4/1/32. Fecha de recuperación: 8 de mayo de 2008.

37. Maki PM, Gast MJ, Vieweg AJ, Burriss SW, Yaffe K. Hormone therapy in menopausal women with cognitive complaints: a randomized, double-blind trial. Neurology 2007;69(13):1322-30.

38. Monterrosa Á, Blumel JE, Chedraui P. Calidad de vida de mujeres en postmenopausia. Valoración con "Menopause Rating Scale" de tres etnias colombianas diferentes. MedUNAB 2009;12(2):80-5.

39. Cerda P, Pino P, Urrutia MT. Calidad de vida en mujeres histerectomizadas. Revista Chilena de Obstetricia y Ginecología 2006;71(3):216-21.

40. Aranibar P. Calidad de vida y vejez. Tesis de maestría. Master en Gerontología Social. Barcelona, España: Universitat de Barcelona; 2005.

41. Velarde E, Ávila, C. Consideraciones metodológicas para evaluar la calidad de vida. Revista de Salud Pública de México 2002;44(5):448-63.

42. Brazier JE, Roberts J, Platts M, Zoellner YF. Estimating a preference-based index for a menopause specific health quality of life questionnaire. Health and Quality of Life Outcomes 2005;3:13 doi: 10.1186/1477-75253-13 [Periódico en línea] Disponible en: http://www. hqlo.com/content/3/1/13. Fecha de recuperación: 14 de mayo de 2008. 